\title{
A supersymmetric type IIB Randall-Sundrum realization
}

M. J. Duff and James T. Liu ${ }^{\text {a) }}$

Randall Laboratory, Department of Physics, University of Michigan, Ann Arbor, Michigan 48109-1120

K. S. Stelle

The Blackett Laboratory, Imperial College, Prince Consort Road, London SW7 2BZ,

United Kingdom

(Received 2 January 2001; accepted for publication 13 February 2001)

We show that an earlier domain wall solution of type IIB supergravity provides a supersymmetric realization of the Randall-Sundrum brane-world, and give its tendimensional interpretation in terms of IIB 3-branes. We also explain how previous no-go theorems are circumvented. In particular, whereas $D=5$ supergravity scalars have $\mathrm{AdS}_{5}$ energy $E_{0} \leqslant 4$ and are unable to support a $D=5$ positive tension brane, our scalar has $E_{0}=8$, and is the breathing mode of the $S^{5}$ compactification. Another essential element of the construction is the implementation of a $Z_{2}$ symmetry by patching together compactifications with opposite signs for their 5-form field strengths. This is thus a IIB analog of a previous $D=5$ 3-brane realization of the Horava-Witten orbifold. A mode-locking phenomenon avoids the appearance of negative energy zero-modes in spite of the necessity of a $D=10$ negative tension brane-source. () 2001 American Institute of Physics. [DOI: 10.1063/1.1372698]

\section{INTRODUCTION}

Our purpose in this paper is first to show that the type IIB domain wall solution of Bremer et al. ${ }^{1}$ provides a supersymmetric realization of the Randall-Sundrum brane-world ${ }^{2,3}$ and second to give its ten-dimensional interpretation in terms of IIB 3-branes.

The idea that our universe may be a 3-brane in a higher-dimensional spacetime has a history going back nearly two decades. ${ }^{4-9}$ More recently, another viewpoint on this basic idea has grown out of the Horava-Witten ${ }^{10,11}$ model for M-theory/heterotic string duality, based upon an $S^{1} / Z_{2}$ orbifold in $D=11$ spacetime. This orbifold construction was later realized in a $D=5$ compactification by a concrete solution to semiclassical M-theory, i.e., $D=11$ supergravity. ${ }^{12,13}$ A key point in this construction was the introduction of flux for the M-theory 4-form field strength $G_{[4]}$ wound around the compact dimensions, which were taken to be a Calabi-Yau 3-fold. The resulting reduced theory is a specific version of matter-coupled $D=5, N=2$ supergravity. This dimensionally-reduced theory has a scalar potential arising from the $G_{[4]}$ flux, which rules out flat space or indeed any maximally symmetric space as a solution to the equations of motion. But this $D=5$ reduced theory readily admits domain wall, i.e., 3-brane, solutions. A natural configuration is a pair of two 3-branes in a $Z_{2}$ symmetric configuration; projecting the fields of this theory into the subspace of $Z_{2}$ invariant configurations then reproduces the Horava-Witten orbifold. As in the original $D=11 / D=10$ discussion, the massless brane-wave excitations of this scenario are not easily deduced by direct analysis of the solution, but one may obtain information about the zero-modes by anomaly inflow arguments. These may either be carried out in $D=10$, leading to the original Hořava-Witten prediction of a $D=10, N=1$ super Yang-Mills $E_{8}$ gauge multiplet residing on each of the two fixed planes of the orbifold, with the resulting structure subsequently reduced to $D=5$, or one may carry out the anomaly analysis directly in $D=5$, yielding more general possibilities for gauge structure. ${ }^{14}$

${ }^{a)}$ Electronic mail: jimliu@umich.edu 
Another theory in which similar constructions can be made is $D=10$ type IIB supergravity. This has a self-dual 5-form field strength that supports the D3-brane, which is the basis for much recent discussion of the Maldacena conjecture, linking string theory in the near-horizon region of the D3-brane to a Yang-Mills theory quantized on the boundary of the associated asymptotic anti de Sitter space, which is the near-horizon limiting spacetime. In the pure supergravity context, relations between $p$-branes in higher dimensions and domain walls arising after dimensional reduction on spheres was developed in Ref. 1, including the case of the D3-brane of type IIB theory.

Meanwhile, another development was brewing. Randall and Sundrum ${ }^{2}$ proposed a simple model of physics on 3-branes embedded in $D=5$ anti de Sitter space, first in a model with two 3-branes, one of positive and one of negative tension. This model was criticized for the apparent danger of nonphysical modes from the negative tension brane, and also because the modulus related to the distance between the two branes gave another parameter needing fixing in any phenomenological analysis. Subsequently, a revision of this scenario was put forward, ${ }^{3}$ in which there was only one 3-brane, of positive tension, essentially obtained from the first scenario by sending the negative tension brane to the Cauchy horizon of anti de Sitter space. The striking result found in this second scenario is that, although the fifth dimension of spacetime is now infinite, the effective gravity theory on the single remaining 3-brane nonetheless has $D=4$ and not $D=5$ leading behavior. The gravitational potential for static sources starts out with a Newtonian $1 / r$, corrected by terms of order $\Lambda^{-1} / r^{3}$, where $\Lambda$ is the $D=5$ cosmological constant. This "binding of gravity" to the 3-brane happens when a $D=5$ spacetime has a warped product structure, with the warp factor, i.e., the factor multiplying the $D=4$ submetric, decreasing as one recedes on either side from the single Randall-Sundrum 3-brane. This corresponds in general terms to the 3-brane acting as a positive-tension source on the right-hand side of the Einstein equations. It was not clear, however, whether this scenario could arise from an explicit solution to a supergravity theory.

Links between the Randall-Sundrum model and supergravity were made in Refs. 15-20. In Refs. 17,18 , the $D=53$-brane solutions to the type II theory presented in Ref. 1 were used to make an analogy to the Randall-Sundrum model. The explicit relation between this construction and the specific Randall-Sundrum model was not fully pinned down, however. This perspective was further elaborated in Refs. 19, 20. Despite the existence of these works, there still seems to be some confusion in the literature as to whether the Randall-Sundrum model can in fact be obtained from an explicit supergravity solution. (Note, however, that the equivalence of the graviton propagator calculated from closed loops of the $N=4$ SCFT in the Maldacena picture and that calculated from tree graphs in the Randall-Sundrum picture was already strongly indicative of a supersymmetric Randall-Sundrum brane-world. ${ }^{21}$ )

Moreover, there are powerful general arguments ${ }^{22-24}$ as to why smooth supersymmetric solutions obtained from $D=5$ gauged supergravity coupled to various combinations of $D=5$ matter cannot reproduce a Randall-Sundrum scenario with binding of gravity to the 3-brane. A key word here is "smooth." Although one might well like to replace the Randall-Sundrum scenario, with its delta-function source, by a smooth solution, experience with domain walls in supergravity (i.e., codimension-one brane solutions) shows them always to be based upon a linear harmonic function in the $d=1$ codimension. In order for such a solution to have a localized energy concentration, i.e., a "brane," some kind of "kink" must be introduced into the linear harmonic function so as to give a location to the domain wall. Thus, the search for a smooth codimension-one solution looks rather unlikely to be successful. (Some rigorous results along these lines have recently been spelled out in Ref. 25. See also Ref. 26.) Moreover, the remainder of the argument of Refs. 22, 23 concerns the general behavior of renormalization group flows between critical points of coupled supergravity-matter potentials. This gives the impression that even if one were to relax the requirement of smoothness, there would be no solution leading to the binding of gravity to the 3-brane.

In this paper, we shall first explicitly obtain the original (kinked) Randall-Sundrum geometry from type IIB supergravity. This follows from the work of Refs. 1, 17, 20. This construction 
makes essential use ${ }^{17}$ of the "breathing mode" of the $S^{5}$ dimensional reduction of type IIB supergravity of Ref. 1 . We shall show why this massive mode escapes the constraints on supersymmetric flows by reason of its transforming in a representation with AdS lowest energy $E_{0}$ $=8>4$, thus falling outside the scope of the analysis of Refs. 22,23 . The breathing-mode solutions, although Kaluza-Klein consistent in a purely bosonic context containing just the breathing mode and gravity, do not really correspond to a pure $D=5$ supergravity theory. The construction retains an essential memory of its $D=10$ type IIB origin. This is particularly so when one considers the superpartners of the breathing mode, which include massive spin two modes that cannot be retained in a consistent truncation to a finite number of $D=5$ fields.

Another memory of $D=10$ supergravity in the supersymmetric realization of the RandallSundrum geometry resides in the $Z_{2}$ symmetry of this geometry. This geometry is very similar to the $Z_{2}$ symmetric configuration of two M-theory 3-branes in $D=5$ that explicitly realizes the Horava-Witten construction as an M-theory brane solution. ${ }^{12,13}$ In the M-theory solution, the $Z_{2}$ symmetry is central to the appearance of the orbifold, and it also plays a critical role in the preservation of unbroken $D=4$ supersymmetry on the brane world-volumes. ${ }^{13}$ The same is true in the double 3-brane type IIB solution that we present as the supergravity realization of the Randall-Sundrum geometry: continuity of the unbroken supersymmetry Killing spinor depends on the way the $Z_{2}$ symmetry is implemented. In particular, in the M-theory case $\mathrm{e}^{13}$ as well as in the type IIB construction, ${ }^{27}$ the constant parameter determining the flux of the relevant underlying form field is $Z_{2}$ odd, and so flips sign upon crossing either of the 3-branes; this flip is crucial for the continuity of the unbroken supersymmetry parameter. Accordingly, in the type IIB case as in the M-theory case, the $D=5$ theory is really obtained from a dimensional reduction on a pair of Kaluza-Klein ansätze, one on each side of the $Z_{2}$ symmetric spacetime. Although this construction requires the presence of brane sources for the form-field flux, it is natural in the context of the higher-dimensional M- or type IIB theory. This split ansatz, however, means that it is much less natural to view the geometry as arising in a single $D=5$ theory.

Having shown how to obtain the Randall-Sundrum model from type IIB supergravity, we next set out to study the brane-wave oscillations of the solution. This analysis is quite natural in the type IIB analog of the M-theory $Z_{2}$ symmetric double 3-brane construction. ${ }^{12,13}$ Although, as in Ref. 2, this configuration involves both a positive and a negative tension brane, thus leading to concerns about negative energies, we show that there is a "mode-locking" phenomenon that reduces the zero-modes to just one (positive energy) $D=4, N=4$ Maxwell multiplet in the case of one singly charged brane. This happens because the $Z_{2}$-odd modes turn out to be nonzero modes constrained to be related to Kaluza-Klein massive modes by the Bianchi identities for the type IIB 5 -form field strength $H_{[5]}$ and for the gravitational curvature. Thus, one does not have to make an explicit projection by hand into a $Z_{2}$-invariant subspace of fields: this projection happens spontaneously, by normal Kaluza-Klein dynamical mechanisms freezing out massive Kaluza-Klein modes. The type IIB models considered here have the great advantage that one can carry out more of the Kaluza-Klein analysis explicitly than in the analogous discussion of M-theory reduced on

Calabi-Yau spaces. ${ }^{12,13}$ But it is to be expected that an analogous mode-locking mechanism will operate there as well. And in that case, the mode-locking can be expected to lead to a spontaneous appearance of $D=4$ chirality, thus generalizing the appearance of chirality by explicit $Z_{2}$ projection.

\section{SUPERSYMMETRIC DOMAIN WALLS AND RENORMALIZATION GROUP FLOWS}

While there are many ways of representing a metric on anti de Sitter space, perhaps the most natural form of the metric from a domain wall point of view is given in terms of Poincare coordinates,

$$
d s^{2}=e^{-2 g y} \eta_{\mu \nu} d x^{\mu} d x^{\nu}+d y^{2} .
$$

Written in this manner, the Minkowski signature boundary of AdS is reached when $y \rightarrow-\infty$, while the point $y \rightarrow \infty$ is instead a null surface, the AdS Killing horizon. In the AdS/CFT corre- 
spondence, this metric is viewed as the near-horizon geometry of $N$ coincident D3-branes, which is described by $\mathcal{N}=4$ super Yang-Mills living on the boundary. Furthermore, the distance to the boundary is regarded as an energy; from the bulk point of view $y \rightarrow-\infty$ is a flow to the UV, while $y \rightarrow \infty$ is a flow to the IR.

The Randall-Sundrum brane-world is obtained by taking two Poincare patches of AdS, both given by (2.1), and joining them at the brane location $y=0$. The resulting Randall-Sundrum metric has the form

$$
d s^{2}=e^{-2 g|y|} \eta_{\mu \nu} d x^{\mu} d x^{\nu}+d y^{2},
$$

and its geometry gives rise to a localized graviton on the "Planck" brane. Presented as "an alternative to compactification," much has been made of the fact that this scenario binds gravity even though the $y$ direction has an infinite extent. Nevertheless, it is apparent from the form of (2.2) that the Planck brane only lives in a tiny portion of AdS, and that movement away from the brane flows towards the Killing horizon and not towards the Minkowski boundary of AdS. Had one instead chosen to join together the $y<0$ regions of (2.1), the resulting geometry would preserve the vast majority of the original space, including the entire portion of AdS near the boundary. This then would yield a divergent "localization" volume and give rise to a brane of opposite character to the Randall-Sundrum brane, namely one that does not bind gravity.

In fact, the above observation motivated the authors of Ref. 28 to view the Randall-Sundrum geometry as a warped compactification of F-theory on a Calabi-Yau four-fold. In this picture, the warped geometry arises from the presence of D3-branes situated on the elliptically fibered CalabiYau manifold. The five-dimensional Randall-Sundrum universe then corresponds to the noncompact four-dimensional spacetime with the addition of a single $y$ coordinate which provides a preferred slicing of the internal space along flows between separated stacks of D3-branes. One thus sees that the Randall-Sundrum brane itself is not identified with any one of the D3-branes, but is instead viewed as an effective geometry that arises in interpolating between the near-horizon locations of the D3-branes. In terms of the parametrization in (2.2), the D3-branes are located at the horizons, $y= \pm \infty$, and the apparent infinite extent of the $y$ coordinate is simply a result of the warping of the compact space by the D3-branes themselves. The localization of gravity is then explained by the compactness of the underlying F-theory construction. Heterotic and M-theory realizations based on warped Calabi-Yau compactifications have been examined in Ref. 29.

Returning to a five-dimensional picture, there have been many attempts to explain the Randall-Sundrum scenario from a supersymmetric domain-wall point of view. The advantage of this approach is that one can generally ignore the added complications of the compactification of the underlying IIB theory, and instead focus only on brane constructions in the resulting $D=5$ gauged supergravity theory. However, as we emphasize below, it is important to realize that there is no reason (other than simplicity) to expect that the relevant degrees of freedom lie only in the massless supergravity sector. In fact, as emphasized in Refs. 22, 23, massless gauged supergravity precludes the localization of gravity on a brane. Thus massive fields are a necessity.

For the Randall-Sundrum picture to be realistic, where the Planck brane is a dynamical object, it would have to be supported by bulk scalar fields. Thus, in the language of bulk renormalization group flow, we seek a brane solution with stable flows to AdS critical points in the IR on both sides of it. This approach has been studied extensively in both the AdS/CFT ${ }^{30,31}$ and brane-world ${ }^{22,23,32,33}$ pictures, with considerable overlap. Nevertheless, the distinction between flows of massless and massive scalars has not always been made clear, so we wish to do so below.

Since we demand that the flow away from the brane is towards an AdS background, the scalars must reach some fixed values corresponding to a critical point in the potential. Then, independent of any specific model, at that point, we may expand the scalars about their fixed values. However before doing so, it is worth realizing that representations in AdS differ from those in a flat background.

Recall that, for $\mathrm{AdS}_{5}$, general representations of $S U(2,2)$ may be labeled by $D\left(E_{0}, j_{1}, j_{2}\right)$ where $E_{0}$ is the lowest energy (which may be given in terms of the natural mass scale of the AdS 
background). For scalars, $D\left(E_{0}, 0,0\right)$, unitarity requires $E_{0} \geqslant 1$ with $E_{0}=1$ corresponding to the singleton representation. General unitarity bounds for $S U(2,2)$ as well as for the $S U(2,2 \mid N / 2)$ superalgebras have been obtained in Refs. 34-39 (see also Ref. 40). For a scalar field in $\operatorname{AdS}_{5}$, the mass is given in terms of $E_{0}$ by $m^{2}=E_{0}\left(E_{0}-4\right)$, so that "massless" scalars in fact correspond to $E_{0}=4$. Of course, negative mass squared is not to be feared in an AdS background, provided the Breitenlohner-Freedman bound ${ }^{41}$ is satisfied. For this case it corresponds to $m^{2} \geqslant-4$, which is saturated for $E_{0}=2$.

To be specific, we now consider the case of a brane supported by a single scalar coupled to gravity, where the Lagrangian takes the form

$$
e^{-1} \mathcal{L}=R-\frac{1}{2} \partial \phi^{2}-V(\phi) .
$$

While one may generalize by including more scalars, this single scalar example is sufficient to bring out our conclusion. The resulting equations of motion have the form

$$
R_{M N}=\frac{1}{2} \partial_{M} \phi \partial_{N} \phi+\frac{1}{3} g_{M N} V(\phi), \quad \nabla^{2} \phi=\partial_{\phi} V(\phi) .
$$

Note that we do not insist that (2.3) necessarily originates from a supersymmetric theory. However, in many cases we are of course interested in supersymmetry. This suggests the identification of a putative "superpotential" $W(\phi)$ with

$$
V=\left(\partial_{\phi} W\right)^{2}-\frac{2}{3} W^{2},
$$

and the putative "transformations,"

$$
\delta \psi_{\mu}=\left[\nabla_{\mu}-\frac{1}{6 \sqrt{2}} W \gamma_{\mu}\right] \epsilon, \quad \delta \lambda=\frac{1}{2}\left[\gamma \cdot \partial \phi+\sqrt{2} \partial_{\phi} W\right] \epsilon .
$$

Identification of the above transformations with those of an actual supergravity theory requires some care. ${ }^{42}$ In particular, from an $N=2$ (i.e., minimal supersymmetry in $D=5$ ) point of view, the field $\phi$ may reside in either a vector, tensor or hypermatter multiplet, with possibly different forms of coupling to the fermions. In all cases, the fields $\left(g_{\mu \nu}, \psi_{\mu}\right)$ and $(\phi, \lambda)$ would be part of a (not necessarily consistent) truncation of the actual supergravity theory.

As emphasized previously in discussions of holographic renormalization group flows, the equations of motion following from a domain-wall ansatz take on a simple form. Starting with the metric

$$
d s^{2}=e^{2 A(y)} \eta_{\mu \nu} d x^{\mu} d x^{\nu}+e^{2 B(y)} d y^{2},
$$

one obtains the following equations:

$$
\begin{gathered}
A^{\prime 2}=\frac{1}{24} \phi^{\prime 2}-\frac{1}{12} e^{2 B} V, \quad A^{\prime \prime}-A^{\prime} B^{\prime}=-\frac{1}{6} \phi^{\prime 2}, \\
\phi^{\prime \prime}+\left(4 A^{\prime}-B^{\prime}\right) \phi^{\prime}=e^{2 B} \partial_{\phi} V,
\end{gathered}
$$

where primes denote $y$ derivatives. The first two equations were obtained by combining components of the Einstein equation. Note that the three equations are not all independent, and we find it convenient to focus only on the first two.

In codimension-one, the second metric factor $e^{2 B}$ is redundant, and may be removed by defining a new coordinate $\tilde{y}=\int e^{B} d y$ (keeping in mind that explicit domain wall solutions often have a simpler form when presented in terms of a metric with the $e^{2 B}$ factor). We proceed by setting $B=0$, so the equations resulting from (2.8) take the form 


$$
A^{\prime \prime}=-\frac{1}{6} \phi^{\prime 2}, \quad A^{\prime 2}=\frac{1}{24} \phi^{\prime 2}-\frac{1}{12} V, \quad \phi^{\prime \prime}+4 A^{\prime} \phi^{\prime}=\partial_{\phi} V .
$$

As emphasized in Refs. 31, 30, the first of these equations indicates that $A^{\prime \prime} \leqslant 0$, with saturation of the inequality corresponding to sitting in the pure AdS vacuum. For the present case, this has the consequence that the function $A(y)$ must be concave-down, which is in fact exactly what is needed to support a "kink-down" (i.e., positive tension) Randall-Sundrum brane of the form (2.2) with a continuous metric function.

To study the behavior of the flow to the IR fixed point, we may expand about the fixed value, $\phi_{*}$, of the scalar. To quadratic order, the potential then has the form

$$
V=-12 g^{2}+\frac{1}{2} m^{2}\left(\phi-\phi_{*}\right)^{2}+\cdots,
$$

where the constant factor is chosen to give the conventional normalization of the AdS curvature,

$$
R_{M N P Q}=-g^{2}\left(g_{M P} g_{N Q}-g_{M Q} g_{N P}\right) \text {. }
$$

While in some cases $g$ may coincide with the coupling constant of gauged supergravity, we only take it to parametrize the AdS background at the specific fixed point in which we are interested.

We now insert (2.10) into the second equation of (2.9) to find that $A(y) \approx \pm g y$, at least up to linear order in $\phi$. Thus we recover the expected linear behavior giving rise to an AdS background. Continuing with the $\phi$ equation of motion, and again working to linear order in $\phi$ (which amounts to making the substitution $A^{\prime} \approx \pm g$ ), we find

$$
\phi^{\prime \prime} \pm 4 g \phi^{\prime}-m^{2} \phi \approx 0
$$

which has in general two solutions:

$$
\phi \approx \phi_{*}+c e^{-E_{0} A(y)}, \text { and } \phi \approx \phi_{*}+c e^{-\left(4-E_{0}\right) A(y)},
$$

where $E_{0}=2+\sqrt{(\mathrm{m} / \mathrm{g})^{2}+4} \geqslant 2$ is given exactly by the mass $/ E_{0}$ relation for a scalar field in AdS space. Additionally, for either flow, the metric function behaves like

$$
A \approx \pm g y-\frac{1}{24}\left(\phi-\phi_{*}\right)^{2}
$$

Finally, this allows us to examine the IR flow, corresponding to the behavior in the direction $A$ $\rightarrow-\infty$. We see that IR stability is ensured for $E_{0}>4$ by taking the second solution of (2.13), while the flow is always unstable for $2 \leqslant E_{0}<4$, and the massless case, $E_{0}=4$, is marginal.

As a result, the above analysis indicates that $E_{0}>4$ is a necessary condition for IR stability, and hence for the construction of a Randall-Sundrum brane. Note, furthermore, that this result was derived without having to appeal to supersymmetry. Thus it holds in general for both BPS and non-BPS flows. However, as we see below, BPS flows impose a further condition on the relative signs of the terms in the superpotential. This powerful and completely general result was in fact present, although hidden in the discussion of Refs. 22, 23. However, in Refs. 22, 23, only scalars residing in massless vector multiplets of $N=2$ gauged supergravity (i.e., the $\mathcal{D}(2,0,0,0)$ representation, where the last value denotes the $U(1)_{r}$ charge) were considered. In particular, the authors of Ref. 22 relied on the relation $\left(\partial_{i} \partial_{j} W\right)_{\mathrm{cr}}=1 / 3 g_{i j} W_{\mathrm{cr}}$ (in our normalization) arising from very special geometry. Such scalars always have $E_{0}=2$, yielding the negative reported result. Curiously, while it may not have been appreciated that scalars in the decomposition of an $N=8$ gauged supergravity multiplet reside in $N=2$ tensor and hypermatter multiplets as well as vector multiplets, such $N=8$ scalars all have $E_{0}=2,3$ or 4 so that they also do not lead to IR stable branes.

Turning now to the case of a supersymmetric flow, it is straightforward to see from (2.6) that the Killing spinor conditions yield the first order equations, 


$$
A^{\prime}= \pm \frac{1}{3 \sqrt{2}} e^{B} W, \quad \phi^{\prime}=\mp \sqrt{2} e^{B} \partial_{\phi} W,
$$

for a domain wall preserving exactly half of the supersymmetries. This result may in fact also be derived without using the transformations (2.6), but instead by a traditional BPS argument for finding static minimum energy configurations. ${ }^{30,33}$ Combining both equations gives rise to a holographic renormalization group flow,

$$
\frac{d \phi}{d A}=-6 \frac{\partial_{\phi} W}{W}
$$

consistent with the second order equations (2.8). In this case, we expand the superpotential as

$$
W= \pm 3 \sqrt{2} g\left(1+\frac{1}{12} \lambda\left(\phi-\phi_{*}\right)^{2}+\cdots\right) \text {, }
$$

corresponding to the potential (2.10), provided $\lambda$ is identified with either $E_{0}$ or $4-E_{0}$. Note that this introduces a two-fold ambiguity. However this is in fact somewhat artificial, since knowledge of the actual supersymmetric theory would fully determine the superpotential (but see, e.g., Ref. 43 for a discussion on the relation between $V$ and $W$ without supersymmetry). In contrast to (2.13), the supersymmetric flow condition, (2.16), gives only a single approach to the fixed point,

$$
\phi \approx \phi_{*}+c e^{-\lambda A(y)}
$$

As a result, for a BPS flow, not only do we require $E_{0}>4$, but also we learn from the above analysis that $\lambda=4-E_{0}$ must be negative in the superpotential (2.17). The requirement of $\lambda<0$ was previously noted in Ref. 22.

This connection between $E_{0}$ and the behavior of a scalar field in AdS was initially made in investigations of the Maldacena conjecture, ${ }^{44,45}$ where $E_{0}$ was related to the conformal dimension of appropriate operators on the CFT side of the AdS/CFT conjecture. In this case, (2.12) taken with exact equality is simply the massive scalar equation in the reference AdS background (2.1). This in itself highlights the similarity between the brane-world scenario and the AdS/CFT conjecture. In some sense, the Randall-Sundrum brane, being inserted at some fixed location in AdS, cuts off the flow to the UV and hence may be described by a Maldacena CFT cut off at some energy scale related to the location of the brane.

\section{BREATHING MODE DOMAIN WALLS AND THE BRANE-WORLD}

Based on the preceding analysis, it is clear that consideration of the massless sector of $(N$ $=2,4$ or 8 ) gauged supergravities alone does not lead to realistic brane-world configurations. However, for a five-dimensional model originating from IIB theory, many other degrees of freedom may come into play. While round $S^{5}$ compactifications of IIB supergravity yield $N=8$ gauged supergravity at the massless level, this is also accompanied by a Kaluza-Klein tower of massive states. In general, consistent truncations of sphere reductions are a delicate matter. ${ }^{46,47}$ However it is consistent to include the breathing mode $\varphi$ in the truncation: although it lives in a massive supermultiplet, it is nevertheless a gauge singlet. Domain walls supported by the breathing mode have been investigated in Refs. 1, 17, 20, and have recently been suggested as possible realizations of the brane-world scenario. Note that we use $\varphi$ to denote the breathing mode rather than $\phi$, in order to emphasize that it is distinct from the $D=10$ dilaton of the type IIB theory.

To make a connection with the Randall-Sundrum model, we examine type IIB string theory compactified on $S^{5}$. This sphere reduction, with the inclusion of a single squashing mode along with the breathing mode, was investigated in Ref. 1. Focusing only on the scalar modes, the resulting five-dimensional Lagrangian is

$$
e^{-1} \mathcal{L}_{5}=R-\frac{1}{2} \partial \widetilde{\varphi}^{2}-\frac{1}{2} \partial \widetilde{f}^{2}-V(\widetilde{\varphi}, \widetilde{f})
$$


The scalar potential has the form

$$
V(\widetilde{\varphi}, \tilde{f})=8 m^{2} e^{(10 / \sqrt{15}) \tilde{\varphi}}+e^{(4 / \sqrt{15}) \tilde{\varphi}}\left(\mu^{2} e^{(6 / \sqrt{10}) \tilde{f}}-R_{4} e^{(1 / \sqrt{10}) \tilde{f}}\right),
$$

where the constants $\left(m, \mu, R_{4}\right)$ are parameters of the compactification. ${ }^{1}$

While this potential may now be expanded in the form of Eq. (2.10), it is perhaps more enlightening to first express it in the form of a "superpotential" according to (2.5). We find

$$
W=2 \sqrt{2} m e^{(5 / \sqrt{15}) \tilde{\varphi}}-e^{(2 / \sqrt{15}) \tilde{\varphi}}\left(\sqrt{2} \mu e^{(3 / \sqrt{10}) \tilde{f}}+\frac{R_{4}}{2 \sqrt{2} \mu} e^{-(2 / \sqrt{10}) \tilde{f}}\right) .
$$

Note that there is a slight sign ambiguity in inverting (2.5); here we have chosen the signs so that $W$ has a critical point at

$$
e^{(3 / \sqrt{15}) \tilde{\varphi}_{*}}=\frac{\mu}{2 m}\left(\frac{R_{4}}{6 \mu^{2}}\right)^{3 / 5}, \quad e^{(5 / \sqrt{10})} \tilde{f}_{*}=\frac{R_{4}}{6 \mu^{2}},
$$

corresponding to that of $V$ as well. Expansion of $W$ then gives

$$
W=-3 \sqrt{2} m\left(\frac{\mu}{2 m}\right)^{5 / 3}\left(\frac{R_{4}}{6 \mu^{2}}\right)\left[1-\frac{1}{3}\left(\widetilde{\varphi}-\tilde{\varphi}_{*}\right)^{2}+\frac{1}{2}\left(\tilde{f}-\tilde{f}_{*}\right)^{2}+\cdots\right] .
$$

A comparison with (2.17) then demonstrates explicitly that the breathing mode $\widetilde{\varphi}$ has $E_{0}=8$ while the squashing mode $\widetilde{f}$ has $E_{0}=6$. Curiously, the two modes enter with opposite signs in $W$. While this $N=8$ symmetric critical point is indeed a minimum of the potential, it is only a saddle point of $W$.

The consequences for the resulting supersymmetric flow were investigated in the previous section. For supersymmetric flows, this critical point is IR stable for the breathing mode, while it is unstable for the squashing mode. This indicates explicitly that simply having a domain wall supported by a scalar with $E_{0}>4$ may be insufficient to ensure the stability of a supersymmetric Randall-Sundrum configuration. Nevertheless, we have now seen why use of the massive breathing mode of sphere reductions has been successful in constructing brane-world domain walls, ${ }^{17,20}$ avoiding the limitations on supersymmetric flows presented in Refs. 22, 23.

To proceed, we now truncate out the squashing mode by setting $\widetilde{f}=0$ and $R_{4}=6 \mu^{2}$ $=(6 / 5) R_{5}$. After dropping tildes, the resulting potential for the breathing mode is simply

$$
V(\varphi)=8 m^{2} e^{(10 / \sqrt{15}) \varphi}-R_{5} e^{(4 / \sqrt{15}) \varphi},
$$

and has an AdS minimum at

$$
e^{(6 / \sqrt{15}) \varphi_{*}}=\frac{R_{5}}{20 m^{2}}
$$

Here $R_{5}$ is the curvature scalar of the round $S^{5}$, arising from the type IIB Kaluza-Klein ansatz, ${ }^{1}$

$$
d s_{10}^{2}=e^{2 \alpha \varphi} d s_{5}^{2}+e^{2 \beta \varphi} d s^{2}\left(S^{5}\right), \quad H_{[5]}=4 m e^{8 \alpha \varphi} \epsilon_{[5]}+4 m \epsilon_{[5]}\left(S^{5}\right),
$$

where

$$
\alpha=\frac{1}{4} \sqrt{\frac{5}{2}}, \quad \beta=-\frac{3}{5} \alpha .
$$

This also indicates that $m$ is essentially the 5-form flux of the Freund-Rubin compactification. Thus the two parameters $m$ and $R_{5}$ of the five-dimensional potential, (3.6), have their origin in the Kaluza-Klein compactification from ten dimensions. Note that the Kaluza-Klein ansatz (3.8) for 
the $H_{[5]}$ field strength implies that the Freund-Rubin parameter $m$ must be odd under transformations $y \rightarrow-y$. In order for this to be realized as a symmetry of the type IIB theory, this "lower" $D=5$ transformation must be accompanied by an orientation-reversing transformation of $S^{5}$, so that the self-dual structure of $H_{[5]}$ is preserved, but with $m \rightarrow-m$. By the "skew-whiffing theorem," ${ }^{46}$ both orientations have the same (maximal) supersymmetry in the case of $S^{5}$. For any other compactifying 5-manifold the supersymmetries would not match.

For a complete truncation of the sphere compactification down to $D=5$, in which all KaluzaKlein modes except for the breathing mode are discarded, the two parameters $m$ and $R_{5}$ satisfy trivial Bianchi identities, and hence must be constant. In this case only a single combination of the two is actually physical. The constant parameter $R_{5}$ may then be viewed as a necessary dimensionful parameter for measuring coordinate distances on the five sphere (much as one would have to introduce a length scale $L$ for toroidal compactification, where periodic coordinates are identified as $y=y+2 \pi L$ ). The actual invariant (physical) size of the five sphere is then set by the expectation of the breathing mode $\varphi$. To see formally how $R_{5}$ may be scaled away, consider a shift of $\varphi$ along with a scaling of $m$,

$$
\varphi \rightarrow \varphi+\sqrt{\frac{15}{4}} \log \lambda, \quad m \rightarrow m \lambda^{-5 / 4} .
$$

This transformation has the effect of multiplying $R_{5}$ by $\lambda$ in the potential (3.6), so that an appropriate choice of $\lambda$ may be used to scale $R_{5}$ to any desired value. A particularly natural choice would be to set $R_{5}=20 \mathrm{~m}^{2}$, so that the AdS critical point is reached at $\varphi_{*}=0$. From a tendimensional point of view, the transformation (3.10) results in

$$
\begin{gathered}
d s_{10}^{2}=\lambda^{5 / 8}\left[e^{2 \alpha \varphi} d s_{5}^{2}+e^{2 \beta \varphi} \lambda \lambda^{-1} d s^{2}\left(S^{5}\right)\right], \\
H_{[5]}=\lambda^{5 / 2}\left[4 m e^{8 \alpha \varphi} \epsilon_{[5]}+4 m \lambda^{-5 / 2} \epsilon_{[5]}\left(S^{5}\right)\right],
\end{gathered}
$$

which is thus a rescaling of $S^{5}$ combined with a $D=5$ "trombone" symmetry.

However, as we will discuss in the following section, if one no longer truncates out the additional Kaluza-Klein modes, then both $m$ and $R_{5}$ no longer need to be taken as constant. In this case, attempts to scale away $R_{5}(x)$ would result in a dynamical scaling by $\lambda(x)$. In this sense one simply trades one parameter for another, and cannot fully eliminate $R_{5}$. With this in mind, we maintain both parameters $m$ and $R_{5}$ in the solution below.

Breathing-mode domain wall solutions follow by making the standard ansatz (2.7) and by solving the resulting equations (2.8). As mentioned above, keeping two independent factors in the ansatz, $A(y)$ and $B(y)$, is redundant. For $B=0$, the solution was presented in Ref. 17, while it was originally presented in Ref. 1 with a different choice of coordinates. The advantage of the original choice is its highlighting of a linear harmonic function as a natural feature of codimension-one $p$-brane solutions. This solution has the basic form ${ }^{1}$

$$
e^{-(7 / \sqrt{15}) \varphi}=H, \quad e^{4 A}=e^{-B}=\widetilde{b}_{1} H^{2 / 7}+\widetilde{b}_{2} H^{5 / 7}, \quad H=e^{-(7 / \sqrt{15}) \varphi_{0}}+k y,
$$

where

$$
\widetilde{b}_{1}=\eta_{1} \frac{28 m}{3|k|}, \quad \tilde{b}_{2}=\eta_{2} \frac{14 \sqrt{5 R_{5}}}{15|k|} .
$$

Here $\eta_{1,2}= \pm 1$ are in general independent choices of signs for the solution. For our purposes they are fixed by requiring an appropriate AdS limit for $\varphi \rightarrow \varphi_{*}$. This gives $\eta_{2}=-\eta_{1}$ and $\eta_{1}$ chosen so that $e^{4 A}>0$ in order for the metric to be real at a given initial value of $y$.

The linear harmonic function $H$ is restricted to be nonnegative. Examination of the solution indicates that the AdS horizon is located at $H=H_{*} \equiv e^{-7 \varphi_{*} / \sqrt{15}}$, where $e^{4 A}$ vanishes. For initial $H>H_{*}$ the five-dimensional space asymptotically flattens out as $H \rightarrow \infty$, with a corresponding limit for the scalar field $\varphi \rightarrow-\infty$, yielding an asymptotically vanishing scalar potential. This case 
is the second branch of Ref. 17, where it was referred to as a hybrid type II and dilatonic domain wall. On the other hand, for initial $H<H_{*}$, the solution soon runs into a singularity at $H=0$. Note, however, that if one starts with a solution with $H>H_{*}$ initially and signs $\eta_{1,2}$ chosen so as to make $e^{4 A}>0$ initially, but then follows the evolution of $H$ within the spacetime through the $H=H_{*}$ horizon, the metric in the region with $H<H_{*}$ becomes complex, so one should really treat the region below the horizon using different, appropriately chosen coordinates. Both the $H>H_{*}$ and $H<H_{*}$ cases have a natural interpretation in the lifting of (3.12) to ten dimensions. In the IIB theory, (3.12) lifts directly to the geometry of $N$ coincident D3-branes with total charge $\tilde{k}$ $=m\left(20 / R_{5}\right)^{5 / 2}$. ${ }^{1}$ The two regions $H \gtrless H_{*}$ then correspond to the regions either "outside" or "inside" the D3-brane horizon. This furthermore demonstrates that the first, $H>H_{*}$, case is nothing but the conventional near-horizon limit occurring prominently in the Maldacena conjecture. The second, $H<H_{*}$, case is unphysical as it stands, however, as it sees a different region of the D3-brane geometry containing a singularity.

Neither case by itself provides a suitable framework for a Randall-Sundrum configuration. While in one direction one may reach an AdS horizon, in the other direction one will either run into a singularity or on out into unbounded flat space. One obvious possibility for obtaining an asymptotically AdS space on both sides of a domain wall is to reflect the solution at $y=0$, imposing thus a $y \rightarrow-y Z_{2}$ symmetry. The resulting two-sided domain wall, supported by an absolute value kink in the linear harmonic function,

$$
H=e^{-(7 / \sqrt{15}) \varphi_{0}+k|y|,}
$$

was in fact how the solution was originally presented in Ref. 1. The presence of such a kink is rather natural for a codimension-one object. Supergravity $p$-brane solutions are generally supported by $\delta$-function sources at the locations of the branes themselves, and this remains true for domain walls. Passing through a domain wall, one jumps through a sheet of charge, and this jump in charge manifests itself in a change in the slope of the linear harmonic function. A priori, the slope could take any values on the two sides of the domain wall, but clearly the $Z_{2}$ symmetric jump from $k$ to $-k$ is a natural configuration. We shall see that this configuration is distinguished also by preserving unbroken supersymmetry.

For either the plain unkinked (3.12) or the kinked (3.14) solution, the slope $k$ may be scaled away by taking $y \rightarrow y /|k|$ and $x^{\mu} \rightarrow x^{\mu}|k|^{1 / 4}$. This explains why the apparent domain wall charge $k$ is not directly related to lifted quantities such as the D3-brane charge $\tilde{k}$. However, note that this scaling does not eliminate the sign of $k$, thus leaving a distinction between the slope-up and slope-down possibilities. For discussions of multiple domain wall configurations or brane fluctuations, it is more convenient to retain $k$.

If one chooses to restrict the coordinate $y$ in (3.14) to range only over the interval $-y_{0} \leqslant y$ $\leqslant y_{0}$, identifying the points $y_{0}$ and $-y_{0}$, then one obtains a $Z_{2}$ symmetric solution that can serve as the background for a $Z_{2}$ orbifold construction. This orbifold construction is analogous to the treatment of M-theory 3-branes given in Refs. 12, 13 as a brane realization of the Horava-Witten $S^{1} / Z_{2}$ orbifold, and has also been proposed in the Randall-Sundrum context in Ref. 20. The identification of $y_{0}$ and $-y_{0}$ essentially reproduces the original Randall-Sundrum model ${ }^{2}$ with both an attractive and a repulsive brane (if one chooses $k<0$, then the attractive brane is the one located at $y=0$ ). From the five-dimensional point of view, the $y \rightarrow-y Z_{2}$ map is a parity flip. As we have mentioned above, however, this alone is not a good symmetry of the underlying type IIB theory. In order for this transformation to be compatible with the round-sphere compactification of the IIB theory, this $Z_{2}$ transformation must combine the flip in $y$ with an orientation-reversing transformation ${ }^{27}$ of the $S^{5}$. For example, an allowable transformation flips all six of the coordinates transverse to the underlying $D=10 \mathrm{D} 3$-brane. The net effect is to send $m \rightarrow-m$ as well as $y \rightarrow-y$.

This orientation reversal has important consequences for the supersymmetry transformations (2.6), since the superpotential $W$ also flips, $W \rightarrow-W$, under these transformations. Actually, this is what one wants, because if the superpotential were to not to flip in this way, then all supersym- 
metries would be broken by the domain wall, and it would then no longer be BPS. To see this, consider for example the $\delta \lambda$ transformation for the solution (3.12) with the linear harmonic function (3.14). By truncating out the squashing mode from (3.3), one arrives at the breathingmode superpotential:

$$
W=\sqrt{2} m\left[2 e^{(5 / \sqrt{15}) \varphi}-5 \sqrt{\frac{R_{5}}{20 m^{2}}} e^{(2 / \sqrt{15}) \varphi} .\right.
$$

Written as above, this clearly changes sign as $m \rightarrow-m$. On the other hand, If one were to assume instead that $W$ remains invariant, one would find

$$
\begin{aligned}
\partial_{\varphi} W & =\frac{2}{3} \sqrt{30} m\left[e^{(5 / \sqrt{15}) \varphi}-\sqrt{\frac{R_{5}}{20 m^{2}}} e^{(2 / \sqrt{15}) \varphi}\right] \\
& =\frac{\sqrt{30}}{14}|k| H^{-1}\left(\left|\widetilde{b}_{1}\right| H^{2 / 7}-\left|\widetilde{b}_{2}\right| H^{5 / 7}\right) \\
& =-\frac{1}{\sqrt{2}}\left|\varphi^{\prime}\right| e^{-B},
\end{aligned}
$$

where the signs $\eta_{1,2}$ have been chosen to obtain the outside (i.e., $H>H_{*}$ ) AdS solution. Inserting this into (2.6) we would find

$$
\delta \lambda=\frac{1}{2} e^{-B}\left(\gamma^{\bar{y}} \varphi^{\prime}-\left|\varphi^{\prime}\right|\right) \epsilon,
$$

where $\bar{y}$ denotes a local Lorentz index. Because of the absolute value in the linear harmonic function (3.14), $\varphi^{\prime}$ changes sign on opposite sides of $y=0$. Therefore the assumption of an invariant $W$ would leave no possibility of obtaining a Killing spinor that is consistently defined on both sides of $y=0$. If one were to attempt to patch together separate Killing spinors on both sides of $y=0$, in the case of an invariant $W$, the $y \gtrless 0$ projections on the supersymmetry parameter would be into mutually orthogonal components, $\left(1+\gamma^{\bar{y}}\right) \epsilon_{+}=0$ vs $\left(1-\gamma^{\bar{y}}\right) \epsilon_{-}=0$. However, since the superpotential does change sign under the $Z_{2}$, the absolute value in (3.17) is in fact not present, and we accordingly find global Killing spinors of the form $\epsilon=e^{A / 2}\left(1+\gamma^{\bar{y}}\right) \epsilon_{0}$. Similar considerations apply at the location of the second kink in the $Z_{2}$ invariant background. If one expands the theory in modes about this $Z_{2}$ invariant background, keeping only the $Z_{2}$ invariant modes, the resulting theory is equivalent to one defined on an $S^{1} / Z_{2}$ orbifold.

As we have just demonstrated, the domain wall solution is always one half supersymmetric, with or without the absolute value kink. In particular, the $Z_{2}$ orbifolding has not destroyed any further supersymmetry beyond the original half-BPS solution. On the other hand, there is no restoration of supersymmetry either in the presence of a kink. Consider taking a simultaneous limit $k \rightarrow 0$ and $\varphi_{0} \rightarrow \varphi_{*}$. Without the kink, this limit would yield pure AdS, i.e., the D3-brane nearhorizon limit in which full supersymmetry is restored. But with the kink, one obtains instead a $Z_{2}$ symmetric patching of AdS, with a Randall-Sundrum brane located, say, at $y=0$. The presence of the orbifold fixed point prevents the full supersymmetry from being restored. However, this is fully expected when a domain wall is present. Although the $Z_{2}$ symmetrization introduces an absolute value into functions, the Killing spinor equations are of first order, and so do not see any $\delta$-function singularities. As long as the conditions (2.15) are satisfied, the solution remains supersymmetric.

Of course the second order equations of motion will see the $\delta$-function brane source. For the solution (3.14), we find that the extra source terms at $y=0$ are 


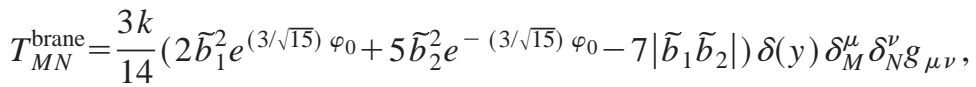

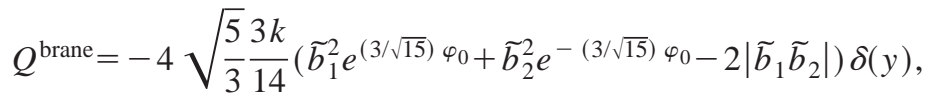

assuming $\widetilde{b}_{1} \widetilde{b}_{2}<0$ as indicated above. These "brane sources", enter in the equations of motion as

$$
R_{M N}-\frac{1}{2} g_{M N} R=T_{M N}^{\varphi}+T_{M N}^{\text {brane }}, \quad \nabla^{2} \varphi=\partial_{\varphi} V(\varphi)+Q^{\text {brane }},
$$

where

$$
T_{M N}=\frac{1}{2}\left(\partial_{M} \varphi \partial_{N} \varphi-\frac{1}{2} g_{M N} \partial \varphi^{2}\right)-\frac{1}{2} g_{M N} V(\varphi)
$$

Depending on the sign of $k$, the branes have either positive or negative energy density. However, in both cases the relation between charge and tension is the same, so the branes may be stacked up in BPS configurations.

We have thus seen that the kinks at the brane locations have different consequences for supersymmetry and for the equations of motion. Since the supersymmetry variations and Killing spinor conditions are of first order, the kinks give rise to possibly discontinuous quantities, but no $\delta$-function singularities. On the other hand, the equations of motion will be sensitive to the additional $\delta$-function sources. Although one may view the equations of motion as a composition of two supersymmetries, there is no contradiction in the presence and absence of the $\delta$-function terms since the Killing spinor equations only give rise to a subset of the full equations of motion. To see this consider again for simplicity the $\delta \lambda$ transformation (2.6),

$$
\delta \lambda=\frac{1}{2}\left[\gamma \cdot \partial_{\phi}+\sqrt{2} \partial_{\varphi} W\right] \epsilon=\frac{1}{2} e^{-B}\left[\varphi^{\prime} \gamma^{\bar{y}}+\sqrt{2} e^{B} \partial_{\varphi} W\right] \epsilon .
$$

Partial breaking of supersymmetry then demands the BPS condition $\varphi^{\prime}=-\sqrt{2} e^{B} \partial_{\varphi} W$, relating the scalar to its potential. One may of course choose the other sign if so desired. However this is a global choice, and must be consistent in all patches of space. Similarly, vanishing of the gravitino relates the metric to the scalar potential, $A^{\prime}=e^{B} W / 3 \sqrt{2}$, as given in (2.15). Now consider deriving the second order $A^{\prime \prime}$ equation of motion by taking a derivative of $e^{-B} A^{\prime}$,

$$
e^{-B}\left(A^{\prime \prime}-A^{\prime} B^{\prime}\right)=\frac{1}{3 \sqrt{2}} W^{\prime} .
$$

For a continuous $W$, one simply uses the chain rule, $W^{\prime}=\partial_{\varphi} W \phi^{\prime}$, and substitutes in the $\varphi^{\prime}$ equation to arrive at the $A^{\prime \prime}$ equation of motion given in (2.8). However, the assumption of a continuous $W$ is actually too strong. For the $Z_{2}$ invariant case, where $W$ changes sign at the brane (say at $y=0$ ), one would also pick up a source term upon differentiating, resulting in

$$
A^{\prime \prime}-A^{\prime} B^{\prime}=-\frac{1}{6} \varphi^{\prime 2}+\frac{\sqrt{2}}{3} e^{B} W \delta(y)
$$

Thus, while supersymmetry implies most of the equations of motion, it does not in fact determine all of them. In fact, for higher codimension branes, there is even more slack between the BPS conditions and the equations of motion. The harmonic function condition, of primary importance in brane constructions, is generally a consequence of the equations of motion, and not supersymmetry. ${ }^{48,49}$ 


\section{D3-BRANES AND THE WORLD IN TEN DIMENSIONS}

Until now we have focused almost exclusively on the five-dimensional viewpoint of the Randall-Sundrum scenario. Since the breathing-mode domain wall has its origins in the $S^{5}$ compactification of IIB theory, it has a natural interpretation in terms of IIB D3-branes. ${ }^{1}$ Following this connection from the brane-world geometry to breathing-mode branes and then to D3-branes, one is led to a realization of the Randall-Sundrum scenario in terms of IIB theory in an appropriate D3-brane background.

While the lifting of the breathing mode brane to patches of the D3 geometry is straightforward, the resulting configuration has unusual features. Following Ref. 1, lifting of the solution given in (3.12) proceeds by identifying a ten-dimensional Schwarzschild coordinate,

$$
\rho=\sqrt{\frac{20}{R_{5}}} H^{3 / 28}
$$

Using the charge relation $\widetilde{k}=m\left(20 / R_{5}\right)^{5 / 21}$ and the Kaluza-Klein ansatz (3.8), one finds the resulting ten-dimensional metric,

$$
d s_{10}^{2}=\widetilde{b}_{2}^{1 / 2}\left(1-\frac{\widetilde{k}}{\rho^{4}}\right)^{1 / 2} d x_{\mu}^{2}+\left(1-\frac{\widetilde{k}}{\rho^{4}}\right)^{-2} d \rho^{2}+\rho^{2} d \Omega_{5}^{2}
$$

which is that of $N$ D3-branes of total charge $\widetilde{k} \cdot{ }^{8,9}$ A further change of coordinates, $r^{4}=\rho^{4}-\widetilde{k}$, may be performed to transform this into standard isotropic form,

$$
d s_{10}^{2}=\sqrt{\widetilde{b}_{2}} H_{D 3}^{-1 / 2} d x_{\mu}^{2}+H_{D 3}^{1 / 2}\left(d r^{2}+r^{2} d \Omega_{5}^{2}\right),
$$

with a harmonic function $H_{D 3}=1+\widetilde{k} / r^{4}$. Note that the constant $\widetilde{b}_{2}$ may easily be scaled out of the longitudinal coordinates.

For the $Z_{2}$ symmetric configuration, obtained by kinking the linear harmonic function, (3.14), we see that $H$ is a double valued function of $y$. This has the consequence that the lifting relation (4.1) is similarly double valued; opposite sides of the breathing-mode brane lift to identical $\rho$ values. While the orbifold picture corresponds to a single slice of the D3-brane geometry, $\rho$ $\in\left[\rho_{-}, \rho_{+}\right]$, the full circle compactification instead corresponds to two copies of the D3-brane geometry patched together at $\rho_{-}$and $\rho_{+}$. Note that the AdS horizon, located at $H_{*}$, lifts to the D3-brane horizon, located at $\rho_{*}=\widetilde{k}^{1 / 4}$. Thus taking the Randall-Sundrum configuration (kinkdown with $H>H_{*}$ ) and pushing the second brane off to the Cauchy horizon corresponds in ten dimensions to taking two copies of the near-horizon geometry of $N$ D3-branes, and gluing them together at a value $\rho_{0}$ of the Schwarzschild coordinate corresponding to the initial value $H_{0}$ of the linear harmonic function.

For this Randall-Sundrum configuration, it is instructive to "unfold" the doubled metric (4.3) by defining a new radial coordinate $\xi \in\left[-r_{0}, r_{0}\right]$ such that $r=r_{0}-|\xi|$. After scaling out $\widetilde{b}_{2}$ from (4.3), the lifted Randall-Sundrum metric has the form

$$
d s_{10}^{2}=\left(1+\frac{\widetilde{k}}{\left(r_{0}-|\xi|\right)^{4}}\right)^{-1 / 2} d x_{\mu}^{2}+\left(1+\frac{\widetilde{k}}{\left(r_{0}-|\xi|\right)^{4}}\right)^{1 / 2}\left(d \xi^{2}+\left(r_{0}-|\xi|\right)^{2} d \Omega_{5}^{2}\right) .
$$

The positive tension brane is located at $\xi=0$, while the negative tension brane is pushed off to the AdS horizon at $\xi= \pm r_{0}$ (the two values are identified under the $Z_{2}$ orbifolding). As seen explicitly here, this act of patching together two stacks of D3-branes essentially compactifies the sixdimensional space transverse to the branes, and also introduces a curvature discontinuity at $\xi$ $=0$, the location of the patching. Furthermore, this compactification introduces a charge conservation condition, implying that the net D3 charge must vanish. Thus the resulting kink at $\xi=0$ 
must include a stack of $2 N$ negative tension D3-branes, with $-2 N$ units of charge soaking up the $N+N$ units of charge from the two stacks of positive tension D3-branes.

The question arises, however, whether placing this stack of $2 N$ negative tension D3-branes at $\xi=0$ is sufficient for generating the kinked Randall-Sundrum geometry. Furthermore, the reduction of D3-brane tension from $D=10$ to $D=5$ yields the simple result $T_{D=5}=T_{D=10}$. In addition to giving rise to the tension discrepancy pointed out in Ref. 27, it also leaves unexplained how positive $D=5$ tension arises from negative $D=10$ tension. As it turns out, the resolution to both issues is the realization that the $Z_{2}$ orbifolding, or the doubling of spacetime, itself gives rise to a positive tension contribution at $\xi=0$, the location of the kink. Of course, it is easy to see that the net tension has to be positive, as that is what is required to "fold up" or compactify the space transverse to the branes. The resulting picture is one of negative tension D3-branes trapped on a positive tension $Z_{2}$ orbifold plane giving rise to a composite description of the Randall-Sundrum configuration. ${ }^{50}$

By starting with a brane-world scenario on a circle, one obviously obtains a compact KaluzaKlein geometry, corresponding to expanding IIB theory about a $\mathcal{M}^{1,3} \times S_{1} \times S^{5}$. The $S_{1}$ coordinate $y$ lifts to the radial coordinate $\rho$, living in a restricted annular range between the two D3 source shells in a double D3-brane background. Of course there is no surprise in starting with a compact geometry and lifting it to another compact scenario. However, by taking the limit of placing the second brane at the Cauchy horizon of AdS, one effectively decompactifies the original RandallSundrum geometry of Ref. 2 into the picture of Ref. 3. Nevertheless, from a ten-dimensional point of view, this corresponds to simply extending the range of $\rho$ a finite distance so as to reach the doubled D3-brane horizon: the internal space remains compact (at least if the inside-horizon brane cores are disregarded). By smoothing out the patching of the double D3-brane configuration, one presumably obtains a warped compactification with an internal six-manifold in the spirit of Ref. 28.

To complete this D3-brane picture of the brane-world, we present the limit in which the $Z_{2}$ symmetric supergravity solution literally reproduces the Randall-Sundrum configuration of a single positive-tension "kink-down" brane between two patches of anti de Sitter space. ${ }^{3}$ Starting from the $D=53$-brane metric (3.12) with $\tilde{b}_{2}>0, \widetilde{b}_{1}<0, k<0$, we want to take a limit as $k$ $\rightarrow 0_{-}$. However, the inverse power of $k$ in $\tilde{b}_{1}$ and $\tilde{b}_{2}$ (3.13) makes this appear singular. The cure for this is to take a coordinated limit as $k \rightarrow 0_{-}$and $\varphi \rightarrow \varphi_{*}$. We implement this explicitly by taking

$$
e^{-(7 / \sqrt{15}) \varphi_{0}}=\left(\frac{20 m^{2}}{R_{5}}\right)^{7 / 6}+\beta|k|, \quad \beta>0
$$

Note that for $\beta>0$, one has $e^{-(7 / \sqrt{15}) \varphi_{0}}>e^{-(7 / \sqrt{15}) \varphi_{*}}$, i.e., $H_{0}>H_{*}$. Accordingly, for finite $k$ $<0$, the harmonic function $H$ decreases from its value $H_{0}$, reaching the Cauchy horizon value $H_{*}$ at $y=y_{h}$. This is the natural point at which to make an identification $y_{h} \leftrightarrow-y_{h}$, putting the second (negative tension) 3-brane at the horizon. For finite $k$, one thus has a "semi-interpolating soliton" in the sense that one of the asymptotic limits of the solution, but not both, corresponds to a vacuum solution of the theory, in this case the AdS space with asymptotic scalar $\varphi_{*}$. At the Randall-Sundrum brane, however, there is no horizon.

Taking the joint limit defined by (4.5) as $k \rightarrow 0_{-}$, the difference between the two harmonic functions in $e^{2 A}$ partially cancels, giving an expression proportional to $k$, which cancels the $k$ in the denominators of $\widetilde{b}_{1}$ and $\tilde{b}_{2}$. The resulting metric function is then given by

$$
e^{4 A}=4 m\left(\frac{R_{5}}{20 m^{2}}\right)^{5 / 6}(\beta-|y|)=\frac{4}{L}(\beta-|y|),
$$

where $L=m^{-1}\left(20 m^{2} / R_{5}\right)^{5 / 6}$ and the $y$ coordinate remains restricted to a compact range, $|y|$ $<\beta$. This corresponds to the line element 


$$
d s^{2}=\frac{2}{\sqrt{L}}(\beta-|y|)^{1 / 2} \eta_{\mu \nu} d x^{\mu} d x^{\nu}+\frac{L^{2}}{16} \frac{d y^{2}}{(\beta-|y|)^{2}} .
$$

The apparent infinite range of the fifth dimension ${ }^{51}$ is obtained by making a change of variables,

$$
\beta-|y|=\beta e^{-4|\tilde{y}| / L}, \quad x^{\mu}=\left(\frac{L}{4 \beta}\right)^{1 / 4} \widetilde{x}^{\mu},
$$

resulting in the five-dimensional metric,

$$
d s^{2}=e^{-2|\tilde{y}| L} \eta_{\mu \nu} d \widetilde{x}^{\mu} d \widetilde{x}^{\nu}+d \widetilde{y}^{2},
$$

which is literally the Randall-Sundrum solution.,3 This sign of the kink $(k<0)$ thus corresponds to a binding of gravity to the 3 -brane at $y=0$, with a metric corresponding to segments of pure anti de Sitter space everywhere off this brane surface.

In taking the above Randall-Sundrum limit $k \rightarrow 0_{-}, \varphi_{0} \rightarrow \varphi_{*}$, the ten-dimensional coordinate $\rho$ is restricted to a progressively limited range near $\widetilde{k}$, or, equivalently, $r$ is progressively restricted to a range near $r=0$. Thus, from a $D=10$ perspective, the "infinite" Randall-Sundrum scenario ${ }^{3}$ corresponds to shrinking the outer (RS) brane source tightly around the inner horizon brane. Clearly, what is infinite and what is infinitesimal in this subject is frame-dependent.

It is instructive to see in addition the scaling of the "brane sources" (3.18) in the RandallSundrum limit. Taking $k \rightarrow 0_{-}$, we find

$$
T_{M N}^{\mathrm{brane}}=-\frac{24}{L^{2}} \delta(y / \beta) \delta_{M}^{\mu} \delta_{N}^{\nu} g_{\mu \nu}=2 V_{*} \delta(y / \beta) \delta_{M}^{\mu} \delta_{N}^{\nu} g_{\mu \nu}
$$

while $Q^{\text {brane }}=0$. This vanishing of the scalar charge is in fact forced on us since $\varphi$ decouples from the solution in this limit. This brings up a key observation that it is not so much the breathing mode $\varphi$ that supports the brane, but rather $H_{[5]}$ flux corresponding to D3 charge. In addition, it is also the behavior of $H_{[5]}$ flux that saves the BPS condition with $Q^{\text {brane }}=0$; the variation $\delta \lambda$ becomes trivial (as it must for a decoupling scalar), while the gravitino transformation becomes that of pure AdS but with a sign flip $W_{*} \rightarrow-W_{*}$ at $y=0$ (corresponding to a Freund-Rubin compactification with opposite $S^{5}$ orientations). This preservation of supersymmetry further supports the D3-brane origin of the Randall-Sundrum brane-world, via the double 3-brane configuration that we have presented.

The above successful reproduction of the Randall-Sundrum scenario with a "kink-down" (i.e., positive tension) domain wall embedded into $D=5$ anti de Sitter space depends crucially upon use of the breathing mode $\varphi$, which we have shown to transform in a necessary $E_{0}>4$ anti de Sitter representation. Noted as a possibility for a Randall-Sundrum scenario in Refs. 17, 20, this mode escapes the analysis of Refs. 22, 23 because it belongs to a massive spin-two multiplet, and thus does not belong to an intrinsically $D=5$ supergravity theory. This is because the full multiplet of the breathing mode's superpartners cannot be retained in a "consistent" KaluzaKlein reduction, since it involves a massive spin two mode, which never can be kept in a consistent reduction on spheres. ${ }^{52}$ With respect to the $D=5, N=8$ supersymmetry, the breathing mode belongs to a multiplet containing 20 copies of the following sets of fields: 1 spin 2,4 spin 3/2, 26 spin 1,20 spin $1 / 2,15$ spin 0 . With respect to a $D=5, N=2$ decomposition, it belongs to a long massive vector supermultiplet ${ }^{53}$ which is another way of explaining why it escaped the analysis of Refs. 22, 23. Since the breathing mode is an $S O(6)$ singlet, only the inclusion of the breathing mode's nonsinglet superpartners leads to difficulties with Kaluza-Klein consistency; truncation to the purely bosonic theory involving just $D=5$ gravity and the breathing mode is fully consistent. 


\section{MODE LOCKING AND SPONTANEOUS REDUCTION TO AN ORBIFOLD}

The $Z_{2}$ symmetric scenario presented above, with two branes of opposite tension and opposite magnetic charge, corresponding to (3.14), is clearly similar to the brane constructions of HoravaWitten orbifolds in M-theory given in Refs. 12, 13. The analogous type IIB situation has the great advantage that one can work out explicitly many features of the dynamics, whereas the analogous discussions in M-theory reduced on Calabi-Yau 3-folds must necessarily remain rather implicit. Here, we wish to explore further the properties of this $Z_{2}$ symmetric solution, and see to which extent it naturally corresponds to an orbifold compactification.

The orbifold compactification may be viewed as a compactification on a circle with an additional projection of all the fluctuations into $Z_{2}$ even states only. In a Kaluza-Klein spirit, however, one can investigate the possibility of removing the enforced $Z_{2}$ projection, in order to see what the theory does purely of its own accord when compactified about the double 3-brane background. Thus, we start without making any $Z_{2}$ projections, but still shall take the $y$ direction to be a circle. As explained above, from a ten-dimensional point of view, the D3-branes now have no noncompact transverse directions. Thus there is an added cohomology constraint, which demands that there cannot be any nonzero net magnetic charge in the compact transverse space. Unlike general warped compactifications, which allow for additional fields and nontrivial topology, we shall maintain our focus on the round $S^{5}$ and the breathing mode of the compactification. Then, the simplest allowed configuration on the circle is to have a simple pair of 3-branes with opposite magnetic charges. Placing the branes at opposite points on the circle gives rise to a $Z_{2}$ symmetric configuration. However, without imposing the $Z_{2}$ orbifold symmetry, it would appear that the branes are free to move independently. But we shall now demonstrate that this is not the case; instead, there is a mode-locking phenomenon that links the fluctuations of the two 3-branes into a $Z_{2}$ invariant combination.

Consider the $y$ coordinate to be periodic with length $2 l$, making the identification at $y$ $=\rho_{1} \leftrightarrow-\rho_{2}$. For bosonic fields on this circle, one must impose continuity conditions at both the locations of the 3-branes. Demanding continuity of the scalar field $\varphi$ and the metric component $e^{2 A}$ at $y=0$ and also at $y=\rho_{1} \leftrightarrow y=-\rho_{2}$, one has four continuity conditions to satisfy. In this discussion we shall take the overall periodicity length $2 l$ to be fixed, so $\rho_{1}+\rho_{2}=2 l$. From continuity of the scalar field $\varphi$, one simply obtains at $y=0$ that the value $\varphi_{0}$ must be a common limit of $\varphi$ as one approaches the $y=0 \mathrm{RS}$ brane either from the left or from the right. Continuity at $y=\rho_{1} \leftrightarrow-\rho_{2}$ implies continuity of the harmonic function $H$, so one obtains $\left|k_{1}\right| \rho_{2}=\left|k_{2}\right| \rho_{2}$, or, using $\rho_{1}+\rho_{2}=2 l$, that $\left|k_{1} / k_{2}\right|=2 l / \rho_{1}-1$. Imposing as well the periodicity conditions on the metric function $e^{2 A}$ at $y=0$ and $y=\rho_{1} \leftrightarrow-\rho_{2}$, one obtains the continuity conditions

$$
\left|\frac{k_{2}}{k_{1}}\right|=\frac{\left|m_{2}\right|-\sqrt{\frac{R_{5(2)}}{20}} e^{(-3 / \sqrt{15}) \varphi_{0}}}{\left|m_{1}\right|-\sqrt{\frac{R_{5(1)}}{20}} e^{(-3 / \sqrt{15}) \varphi_{0}}}=\frac{\left|m_{2}\right|-\sqrt{\frac{R_{5(2)}}{20}}\left(e^{(-3 / \sqrt{15}) \varphi_{0}}+\left|k_{2}\right| \rho_{2}\right)}{\left|m_{1}\right|-\sqrt{\frac{R_{5(1)}}{20}}\left(e^{\left.(-3 / \sqrt{15}) \varphi_{0}+\left|k_{1}\right| \rho_{1}\right)} .\right.} .
$$

These conditions are solved by matching relations for $m$ and $R_{5}$ between the two regions:

$$
m_{2}=\left(\frac{2 l}{\rho_{1}}-1\right)^{-1} m_{1}, \quad \sqrt{R_{5(2)}}=\left(\frac{2 l}{\rho_{1}}-1\right)^{-1} \sqrt{R_{5(1)}} .
$$

Accordingly, if one now makes a standard soliton-physics ansatz by letting the $Z_{2}$-odd modulus $\rho_{1}$ become dependent upon the $D=4$ coordinates $x^{\mu}$, then upon substitution back into the field equations, one obtains the effective equation for $\rho_{1}\left(x^{\mu}\right)$. Because the oscillations of this coordinate are linked by (5.2) to the Kaluza-Klein ansatz parameters $m$ and $R_{5}$, however, this specific 
modulus has special restrictions on its oscillations. Both $m$ and $R_{5}$ are curvature components, and are thus subject to Bianchi identities. To see this for $m$, consider the Kaluza-Klein ansatz (3.8), together with the Bianchi identity

$$
d H_{[5]}+\frac{1}{2} \epsilon_{i j} F_{[3]}^{i} F_{[3]}^{j} \equiv 0 .
$$

Letting $m \rightarrow m(x)$ and substituting the original ansatz (3.8), one obtains directly a suppression of $m$ fluctuations, $\partial_{\mu} m(x)=0$. For this reason, parameters entering into generalized Kaluza-Klein ansätze like (3.8) have been sometimes been called "nonzero modes." ${ }^{13}$ In order to see the dynamics of such modes in more detail, one should restore the massive Kaluza-Klein modes that are normally set to zero in a compactification. In the case of $m$, this means replacing the ansatz (3.8) by

$$
H_{[5]}=4 m(x) e^{8 \alpha \varphi} \epsilon_{[5]}+4 m(x) \epsilon_{[5]}\left(S^{5}\right)+h_{[5]},
$$

where $h_{[5]}$ represents the fluctuating massive Kaluza-Klein modes. Re-performing the analysis of the Bianchi identity (5.3) for this generalized ansatz, one now shows that a nonvanishing $\partial_{\mu} m$ must be proportional to $\left.\epsilon^{z_{1} z_{2} z_{3} z_{4} z_{5}} \partial_{\left[z_{1}\right.} h_{|\mu|} z_{2} z_{3} z_{4} z_{5}\right]$, where $h_{\mu z_{2} z_{3} z_{4} z_{5}}$ is a Kaluza-Klein massive mode, with mass determined as usual by the inverse radius of the $S^{5}$ internal sphere, i.e., corresponding to the length scale of the $D=5$ anti de Sitter space. Thus, $m(x)$, and hence $\rho_{1}(x)$ are in fact Kaluza-Klein massive modes, and become "frozen out" at energies lower than the AdS scale. Similar considerations apply to the nonzero mode $R_{5}$, which is the Ricci scalar of the internal $S^{5}$ sphere, upon use of the gravitational curvature Bianchi identity. Specifically, in the simple case with Kaluza-Klein massive modes set to zero, if one sets to zero the $D=5$ Bianchi identity $\nabla^{M}\left(R_{M N}-1 / 2 g_{M N} R\right)=0$ and uses the dimensionally reduced field equations, one finds, for $R_{5} \rightarrow R_{5}\left(x^{\mu}\right)$, the constraint $\partial_{\nu} R_{5} \exp (1 / 2 \sqrt{5 / 3} \varphi)-m \partial_{\nu} m=0$, thus locking out the low energy $R_{5}\left(x^{\mu}\right)$ fluctuations as well.

Given that the $Z_{2}$ odd modes are linked via Bianchi identities to massive Kaluza-Klein modes, one expects the theory to settle down into a low energy effective theory that is $Z_{2}$ symmetric. Strictly speaking, all that has been demonstrated above so far is that the $D=4$ derivatives $\partial_{\mu} m, \partial_{\mu} R_{5}$ are locked out at low energies. In order to show that the theory settles down into a $Z_{2}$ symmetric lowest energy configuration, one would need either to analyze in detail the energy functional for the compactified theory, or to study in more detail the equations of motion of the massive modes. It is likely that the analysis of $Z_{2}$ odd modes can only be done fully consistently if one keeps the entire Kaluza-Klein towers of massive states.

However, one can get an idea of the situation that is obtained with non- $Z_{2}$-symmetric configurations if one considers in a little more detail the question of supersymmetry preservation in a patched background with the matching conditions (5.1), (5.2). Locally, in a patch, there is no difficulty in finding a Killing spinor. However, once one declares that the overall compact part of the spacetime is $S^{5} \times S^{1}$, one is required to impose continuity and periodicity conditions both for bosons and for fermions.

In the $Z_{2}$ symmetric configuration of the two 3-branes, we have already demonstrated while discussing the unbroken supersymmetry transformation of Sec. III,

$$
\delta \lambda=\frac{1}{2} e^{-B}\left(\gamma^{\bar{y}} \varphi^{\prime}-\varphi^{\prime}\right) \epsilon,
$$

that there is a consistently defined and continuous unbroken supersymmetry transformation with a $Z_{2}$ even global Killing spinor $\epsilon=e^{A / 2}\left(1+\gamma^{\bar{y}}\right) \epsilon_{0}$. Now consider the form of the broken supersymmetry transformations in the double 3-brane background. As one can see from the supersymmetry algebra the anticommutator $\left\{Q_{\text {broken }}, Q_{\text {preserved }}\right\}$ involves a translation in the fifth coordinate $y$, which is clearly $Z_{2}$ odd. Indeed, the broken supersymmetry parameters will have $Z_{2}$ odd projection conditions. This $Z_{2}$ odd character is canceled, however, in expressions for Goldstone spinor zero modes like (5.5), by the $Z_{2}$ odd character of $\varphi^{\prime}$. Combining the Goldstino expression for 
$y>0$ with the $Z_{2}$ map for $y<0$ amounts to inserting an absolute value sign around $\varphi^{\prime}$ in (5.5), taking a broken supersymmetry parameter for $\epsilon$. Thus, overall, the Goldstino zero mode is $Z_{2}$ even, as it must be in a consistent truncation. Note that the "kink" in the Goldstino expression resulting from (5.5) with the replacement $\varphi^{\prime} \rightarrow\left|\varphi^{\prime}\right|$ corresponds to the sign flip of the superpotential $W$. That $W$ flips without necessarily passing through zero is what allows the Goldstino mode to be normalizable in the present case, thus circumventing the normalizability problems for Goldstinos described in Ref. 54. Overall, the zero modes of the double 3-brane geometry form a single $D=4, N=4$ super Maxwell multiplet.

Now consider what happens if one tries to expand around a non- $Z_{2}$-symmetric configuration of 3-branes. For the Killing spinor itself, one may observe that $\epsilon=e^{A / 2}\left(1+\gamma^{\bar{y}}\right) \epsilon_{0}$ is in fact still continuous and well-behaved in the nonsymmetric case, since the metric function $e^{2 A}$ is by construction matched at the branes. However the situation is different for the candidate Goldstinos. For a non- $Z_{2}$-symmetric configuration the derivative $\varphi^{\prime}$ differs by more than a sign as one crosses a 3-brane: in this case one has $\left|k_{2}\right| \neq\left|k_{1}\right|$, so there is a nonunimodular factor present as well. This prevents one from having continuity both of the unbroken supersymmetry parameter and of the Goldstinos. Thus, although things look locally like one has a BPS configuration with unbroken supersymmetry for a non- $Z_{2}$-symmetric configuration, analysis of the putative zeromode supermultiplets finds them to be inconsistent with the available matching conditions. So, one is led to conclude that only the $Z_{2}$ symmetric configuration has a proper unbroken supersymmetry and zero-mode multiplets transforming correctly with respect to it.

The configuration with globally unbroken supersymmetry should be the proper "vacuum" in this double 3-brane sector of type IIB theory compactified on $S^{5}$. A fuller analysis of this spontaneous reduction to a $Z_{2}$ invariant effective theory on the basis of energy functionals and the equations of motion for the Kaluza-Klein massive modes would be desirable. But it is already clear that this double 3-brane model displays a remarkable spontaneous appearance of an orbifold structure. This happens not by insistent projection into a $Z_{2}$ invariant sector of the theory, but naturally by virtue of the Kaluza-Klein dynamics of the theory.

Our discussion has indicated that the original Randall-Sundrum model ${ }^{2}$ arises naturally when the fifth dimension $y$ direction is taken to be compact, and one may view the model as a system of two D3-branes transverse to the internal $S_{1} \times S^{5}$. From the $D=5$ point of view, there are two branes: one with positive and one with negative tension, constrained by Kaluza-Klein dynamics to live at diametrically opposed points on the circle. While the presence of a negative tension brane might appear troublesome, we have shown that it does not contribute to the naively anticipated negative energy modes; these are nonzero modes and mix with higher Kaluza-Klein massive modes. The negative tension 3-brane has the effect of protecting the spacetime from curvature singularities in the geometry that might reside behind the Cauchy horizon. Of the a priori two independent types of motion of the 3-branes along the $S^{1}$ direction, only the $Z_{2}$ even modes, corresponding to an overall "rotation" of both branes along the circle, localized in the $D=4$ coordinates $x^{\mu}$, correspond to genuine zero modes.

\section{CONCLUSIONS}

We have found that an appropriately constructed D3-brane configuration provides a supersymmetric and dynamically stable Randall-Sundrum scenario. This is achieved in a solution to the $D=10$ type IIB supergravity equations which can be given a $D=5$ interpretation, but is not fully a $D=5$ solution, for it employs an intrinsically massive Kaluza-Klein mode, the $S^{5}$ breathing mode. This mode has AdS energy $E_{0}=8$, satisfying the bound $E_{0}>4$ that is required for an asymptotic approach to AdS space from a downwards-facing warp-factor kink in a RandallSundrum scenario. There is also a $Z_{2}$ flip in the sign of the Freund-Rubin parameter $m$. This is natural enough in a $D=10$ context where $m$ is a field-strength value, but it is less natural from a $D=5$ viewpoint, where $m$ normally would appear as a parameter. We have found, moreover, that although one can decide to exclude the $Z_{2}$ odd modes when expanding the theory around the presented $Z_{2}$ invariant background, and thus reproduce an $S^{1} / Z_{2}$ orbifold reduction, it is not 
actually necessary to make this projection by hand. Bianchi identities for the curvature values entering in the solution relate the $Z_{2}$ odd modes to Kaluza-Klein massive states of the theory, and so they decouple naturally at low energy. Although charge conservation on the circle requires branes to come in oppositely charged pairs, we have seen that one can recover a single brane Randall-Sundrum model by pushing the second brane off to the Cauchy horizon (i.e., by taking $\varphi_{1}=\varphi_{*}$ for the second brane). From the $D=10$ point of view, however, this corresponds to shrinking an outer RS shell of D3 brane tightly around an inner "horizon" D3 brane of opposite charge and tension. Clearly, an important problem is whether this geometry can be realized in a string theory context.

Note added in proof. As this paper was in the final stages of preparation, a very interesting paper appeared ${ }^{55}$ that sheds light on the relationship between constructions such as those of Refs. 12,13 or the present paper and the supersymmetry scheme of Ref. 56, which was otherwise puzzling. In Ref. 55 supersymmetry in orbifolds, and in particular the $D=5$ case of interest here is discussed. In order to obtain a continuous Killing spinor at orbifold singularities (necessary for the Killing equation to be realized everywhere, including at the singular points), Ref. 55 introduces a 5-form "theory of nothing" field strength, which has just a constant as a solution, but allows for this variable to be only piecewise constant. This allows for a $Z_{2}$ sign flip in the prepotential that is critical for having a preserved supersymmetry allowing coupling to supermatter. This sign flip was not made in the discussion of Ref. 56, leading to problems with matter coupling. This difficulty of Ref. 56, and the resolution of Ref. 55 was also investigated in Refs. 57, 58 and independently worked out by Ref. 59 . We anticipate that a fuller Kaluza-Klein treatment of the type IIB theory, including all fermions and making a careful reduction of the type IIB supersymmetry transformations, will show that the $D=5$ supersymmetry realization adopted in Ref. 55 can also be viewed as the natural dimensional reduction of the type IIB theory using a $Z_{2}$ symmetrized ansatz of the type employed in Refs. 12, 13 and the present paper. In particular, we expect that the 5-form "theory of nothing" field introduced in Refs. 58, 55 can be identified with the $D=5$ residue of the type IIB self-dual 5-form field strength.

\section{ACKNOWLEDGMENTS}

We would like to acknowledge stimulating discussions with Shanta de Alwis, Mirjam Cvetic, Marc Henneaux, Renata Kallosh, Neil Lambert, Finn Larsen, Ruben Minasian, Herve Partouche, Chris Pope, Lisa Randall, Hisham Sati and Herman Verlinde. The research of M.J.D. was supported in part by National Science Foundation (NSF) Grant No. PHY-9722090. The research of K.S.S. was supported in part by PPARC under SPG Grant No. 613.

\footnotetext{
${ }^{1}$ M. S. Bremer, M. J. Duff, H. Lü, C. N. Pope, and K. S. Stelle, "Instanton cosmology and domain walls from M-theory and string theory," Nucl. Phys. B 543, 321 (1999).

${ }^{2}$ L. Randall and R. Sundrum, "A large mass hierarchy from a small extra dimension," Phys. Rev. Lett. 83, 3370 (1999).

${ }^{3}$ L. Randall and R. Sundrum, “An alternative to compactification,” Phys. Rev. Lett. 83, 4690 (1999).

${ }^{4}$ K. Akama, "Pregeometry," Lect. Notes Phys. 176, 267 (1982).

${ }^{5}$ V. A. Rubakov and M. E. Shaposhnikov, "Do we live inside A domain wall?,” Phys. Lett. B 125, 136 (1983).

${ }^{6}$ V. A. Rubakov and M. E. Shaposhnikov, "Extra space-time dimensions: Towards a solution to the cosmological constant problem,’ Phys. Lett. B 125, 139 (1983).

${ }^{7}$ G. W. Gibbons and K. Maeda, "Black holes and membranes in higher dimensional theories with dilaton fields," Nucl. Phys. B 298, 741 (1988)

${ }^{8}$ G. T. Horowitz and A. Strominger, "Black strings and $p$-branes," Nucl. Phys. B 360, 197 (1991).

${ }^{9}$ M. J. Duff and J. X. Lu, “The self-dual type IIB superthreebrane," Phys. Lett. B 273, 409 (1991).

${ }^{10} \mathrm{P}$. Hořava and E. Witten, "Heterotic and type I string dynamics from eleven dimensions," Nucl. Phys. B 460, 506 (1996).

${ }^{11}$ P. Horav̌a and E. Witten, "Eleven-dimensional supergravity on a manifold with boundary," Nucl. Phys. B 475, 94 (1996).

${ }^{12}$ A. Lukas, B. Ovrut, K. S. Stelle, and D. Waldram, "The universe as a domain wall," Phys. Rev. D 59, 086001 (1999).

${ }^{13}$ A. Lukas, B. Ovrut, K. S. Stelle, and D. Waldram, “Heterotic $M$-theory in five dimensions," Nucl. Phys. B 552, 246 (1999).

${ }^{14}$ A. Lukas and K. S. Stelle, "Heterotic anomaly cancellation in five dimensions," J. High Energy Phys. 1, 10 (2000).
} 
${ }^{15}$ D. Youm, "Solitons in brane worlds," Nucl. Phys. B 576, 106 (2000).

${ }^{16}$ D. Youm, "Probing solitons in brane worlds," Nucl. Phys. B 576, 123 (2000).

${ }^{17}$ M. Cvetič, H. Lü, and C. N. Pope, "Domain walls and massive gauged supergravity potentials," Class. Quantum Grav. 17, 4867 (2000).

${ }^{18}$ M. Cvetič, H. Lü, and C. N. Pope, "Localised gravity in the singular domain wall background?," hep-th/0002054.

${ }^{19}$ S. P. de Alwis, "Brane world scenarios and the cosmological constant," Nucl. Phys. B 597, 263 (2001).

${ }^{20}$ S. P. de Alwis, A. T. Flournoy, and N. Irges, "Brane worlds, the cosmological constant and string theory," J. High Energy Phys. 101, 27 (2001).

${ }^{21}$ M. J. Duff and J. T. Liu, “Complementarity of the Maldacena and Randall-Sundrum pictures,” Phys. Rev. Lett. 85, 2052 (2000).

${ }^{22}$ R. Kallosh and A. Linde, "Supersymmetry and the brane world," J. High Energy Phys. 2, 5 (2000).

${ }^{23}$ K. Behrndt and M. Cvetič, "Anti-de Sitter vacua of gauged supergravities with 8 supercharges," Phys. Rev. D 61, 101901 (2000)

${ }^{24}$ A. Ceresole and G. Dall'Agata, "General matter coupled $\mathcal{N}=2, D=5$ gauged supergravity," Nucl. Phys. B 585, 143 (2000).

${ }^{25}$ J. Maldacena and C. Nuñez, "Supergravity description of field theories on curved manifolds and a no-go theorem," hep-th/0007018.

${ }^{26}$ M. Wijnholt and S. Zhukov, “'On the uniqueness of black hole attractors,” hep-th/9912002.

${ }^{27}$ P. Kraus, "Dynamics of anti-de Sitter domain walls," J. High Energy Phys. 9912, 11 (1999).

${ }^{28}$ C. S. Chan, P. L. Paul, and H. Verlinde, “A note on warped string compactification,” Nucl. Phys. B 581, 156 (2000).

${ }^{29}$ P. Mayr, "Stringy world branes and exponential hierarchies," J. High Energy Phys. 11, 13 (2000).

${ }^{30}$ K. Skenderis and P. K. Townsend, "Gravitational stability and renormalization-group flow," Phys. Lett. B 468, 46 (1999).

${ }^{31}$ D. Z. Freedman, S. S. Gubser, K. Pilch, and N. P. Warner, "Renormalization group flows from holographysupersymmetry and a $c$-theorem," Adv. Theor. Math. Phys. 3, 363 (1999).

${ }^{32} \mathrm{~K}$. Behrndt and M. Cvetič, "Supersymmetric domain wall world from $D=5$ simple gauged supergravity," Phys. Lett. B 475, 253 (2000).

${ }^{33}$ O. DeWolfe, D. Z. Freedman, S. S. Gubser, and A. Karch, "Modeling the fifth dimension with scalars and gravity," Phys. Rev. D 62, 46008 (2000).

${ }^{34}$ M. Flato and C. Fronsdal, "Representations of conformal supersymmetry," Lett. Math. Phys. 8, 159 (1984).

${ }^{35}$ V. K. Dobrev and V. B. Petkova, "All positive energy unitary irreducible representations of extended conformal supersymmetry," Phys. Lett. B 162, 127 (1985).

${ }^{36}$ I. Bars and M. Gunaydin, "Unitary representations of noncompact supergroups," Commun. Math. Phys. 91, 31 (1983).

${ }^{37}$ M. Gunaydin, "Unitary highest weight representations of noncompact supergroups," J. Math. Phys. 29, 1275 (1988).

${ }^{38}$ M. Gunaydin, D. Minic, and M. Zagermann, "Novel supermultiplets of $S U(2,2 \mid 4)$ and the $A d S_{5} / C F T_{4}$ duality," Nucl. Phys. B 544, 737 (1999).

${ }^{39}$ M. Gunaydin, D. Minic, and M. Zagermann, " $4 \mathrm{D}$ doubleton conformal theories, $C P T$ and IIB strings on $A d S_{5} \times S^{5}$,", Nucl. Phys. B 534, 96 (1998).

${ }^{40} \mathrm{~S}$. Ferrara, M. A. Lledo, and A. Zaffaroni, "Born-Infeld corrections to D3 brane action in $A d S_{5} \times S_{5}$ and $N=4, D$ = 4 primary superfields,' Phys. Rev. D 58, 105029 (1998).

${ }^{41}$ P. Breitenlohner and D. Z. Freedman, "Stability in gauged extended supergravity," Ann. Phys. (N.Y.) 144, 249 (1982).

${ }^{42}$ In this paper, we have not performed a full Kaluza-Klein reduction of the type IIB supersymmetry transformations, so all discussions of supersymmetry here are somewhat schematic. They will be sufficient, however, to determine the necessary $Z_{2}$ behavior for having an unbroken $D=5$ supersymmetry.

${ }^{43}$ J. de Boer, E. Verlinde, and H. Verlinde, “'On the holographic renormalization group,” J. High Energy Phys. 8, 3 (2000).

${ }^{44}$ S. S. Gubser, I. R. Klebanov, and A. M. Polyakov, "Gauge theory correlators from non-critical string theory," Phys. Lett. B 428, 105 (1998).

${ }^{45}$ E. Witten, "Anti-de Sitter space and holography," Adv. Theor. Math. Phys. 2, 253 (1998).

${ }^{46}$ M. J. Duff, B. E. W. Nilsson, and C. N. Pope, “Kaluza-Klein supergravity,” Phys. Rep. 130, 1 (1986).

${ }^{47}$ M. Cvetič, H. Lü, and C. N. Pope, "Consistent Kaluza-Klein sphere reductions," Phys. Rev. D 62, 064028 (2000).

${ }^{48}$ M. J. Duff, R. R. Khuri, and J. X. Lu, “String solitons," Phys. Rep. 259, 213 (1995).

${ }^{49}$ M. J. Duff, J. T. Liu, H. Lü, and C. N. Pope, “Gauge dyonic strings and their global limit,” Nucl. Phys. B 529, 137 (1998).

${ }^{50}$ M. Cvetič, M. J. Duff, J. T. Liu, H. Lü, C. N. Pope, and K. S. Stelle, “Randall-Sundrum brane tensions," hep-th/ 0011167.

${ }^{51}$ As always in anti de Sitter space, the proper distance to the Cauchy horizon is infinite, but the affine parameter along a null geodesic to the horizon is finite.

${ }^{52}$ M. J. Duff, C. N. Pope, and K. S. Stelle, "Consistent interacting massive spin-2 requires an infinity of states," Phys. Lett. B 223, 386 (1989).

${ }^{53}$ The $N=8 \rightarrow N=2$ decomposition of this bulk multiplet in terms of superconformal $N=4, D=4$ boundary supermultiplets was given in Ref. 40.

${ }^{54}$ G. W. Gibbons and N. D. Lambert, "Domain walls and solitons in odd dimensions," Phys. Lett. B 488, 90 (2000).

${ }^{55}$ E. Bergshoeff, R. Kallosh, and A. Van Proeyen, "Supersymmetry in singular spaces," J. High Energy Phys. 10, 33 (2000).

${ }^{56}$ R. Altendorfer, J. Bagger, and D. Nemeschansky, “Supersymmetric Randall-Sundrum scenario,” hep-th/0003117. 
${ }^{57}$ A. Falkowski, Z. Lalak, and S. Pokorski, “Supersymmetrizing branes with bulk in five-dimensional supergravity," Phys. Lett. B 491, 172 (2000).

${ }^{58}$ N. Alonso-Alberca, P. Meessen, and T. Ortin, "Supersymmetric brane-worlds," Phys. Lett. B 482, 400 (2000).

${ }^{59}$ J. T. Liu, R. Minasian, and H. Partouche, unpublished. 